\title{
Vascular Ring; Spectrum of Pathologies and Scenarios for Diagnosis and Management
}

\author{
Mostafa Elhelali, Mohamed-Adel EIgamal, Ahmed Mostafa, Hysam Abdelmohty*, Walid Hassan \\ Cardiothoracic Surgery Department, Faculty of Medicine, Mansoura University, Mansoura, Egypt \\ Email address: \\ hysam.mohty@gmail.com (H. Abdelmoht) \\ ${ }^{*}$ Corresponding author \\ To cite this article: \\ Mostafa Elhelali, Mohamed-Adel Elgamal, Ahmed Mostafa, Hysam Abdelmohty, Walid Hassan. Vascular Ring; Spectrum of Pathologies and \\ Scenarios for Diagnosis and Management. International Journal of Cardiovascular and Thoracic Surgery. Vol. 6, No. 6, 2020 , pp. 70-74. \\ doi: $10.11648 /$ j.ijcts.20200606.12
}

Received: June 27, 2020; Accepted: July 9, 2020; Published: December 4, 2020

\begin{abstract}
Background: Vascular rings represent approximately 1\% of all congenital cardiac anomalies, with Edward's classification being the first to outline them into a complete or partial vascular ring. A Complete ring is a combination of patent vessels, atretic vascular segments or ligamentous structures. Methods: We reviewed patients records from our Pediatric Cardiac Surgery Unit, over 10 years. We encountered 63 patients from January 2009 to January 2019. Our patients were 57 (90.5\%) complete vascular rings, 5 (7.9\%) pulmonary artery slings, and $1(1.6 \%)$ patient with innominate artery compression. Results: The age of our Patients ranged from 2 to 57 months (mean $16.25 \pm 1.3 \mathrm{~m}$ ), weight ranges from $3 \mathrm{k}$ to $26 \mathrm{k}$ (mean $9.87 \pm 0.8 \mathrm{~kg})$. Males were $38(60.3 \%)$ and male: female ratio was 1.5:1. In our series various types of vascular rings were encountered; Double Aortic Arch was the right dominant arch variant in $25(53.2 \%)$ and left arch dominant in 7 (14.9\%) whilethe co-dominant variant was $15(31.9 \%)$. Fifty-seven cases of vascular rings operated through left posterolateral thoracotomy. Fivecases of pulmonary artery sling (7.9\%) operated by median sternotomy and one case (1.6\%) of innominate artery compression. No reported intraoperative mortality in our study. Conclusion: Vascular ring is a rare anomaly that needs suspicion for diagnosis. The operative strategy relies on extensive dissection and division of the non-dominant arch and division of the ligamentum, freeing all fibrous bands between the arch and esophagus and trachea. For augmented results; we need collaboration from pediatricians and pulmonologists who must be aware of such anomalies.
\end{abstract}

Keywords: Anomaly, Arch, Sling, Ring, Compression

\section{Background}

Under the term vascular rings; a constellation of anomalies related to defective development of the branchial arches. They present as an abnormal position, doubling, interrupted anomalous origin of the aortic arch or its branches [1]. They encircle the aero digestive tracks within the mediastinum; thus presentation is wheezy chest, recurrent pneumonia, stridor, troubled feeding or choking, and failure to thrive. This quite uncommon subcategory of cardiac anomalies accounts for $1 \%$ or less with equal incidence in both sexes. They may present alone or are associated with other major anomalies of the heart like Ventricular septal defect, Fallot's tetralogy, or trans-positioning of great vessels [1].

\section{Methods}

\subsection{Study Design}

It is a retrospective observational longitudinal study on vascular rings management at pediatric cardiac surgery unit. We reviewed our patients' records over a period of 5years. Mansoura University Children Hospital is a high volume, referral institution sees from 8-12 of these patients per year. We encountered 63 patients subjected to surgery from January 2015 to January 2020. Our patients were 57 (90.5\%) complete vascular rings, $5(7.9 \%)$ pulmonary artery slings, and 1 patient $(1.6 \%)$ with innominate artery compression. About one-quarter of these patients $(23.8 \%)$ have a chromosome abnormality. The ethical committee had approved both the records review process and the study plan. 
The classification adopted and used generally classifies vascular rings into 2 main types:

1. Complete Rings. [1, 2]

Double Aortic Arch; (Right arch dominant. -Left arch dominant. Balanced). Right arch/Left ligamentum
(Retroesophageal Lt subclavian. -Mirror image branching.). 2. Incomplete Rings

Innominate artery compression syndrome.

Pulmonary artery sling.

Left arch/aberrant Right subclavian artery.

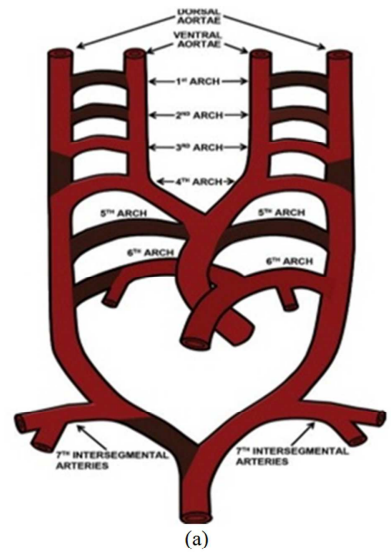

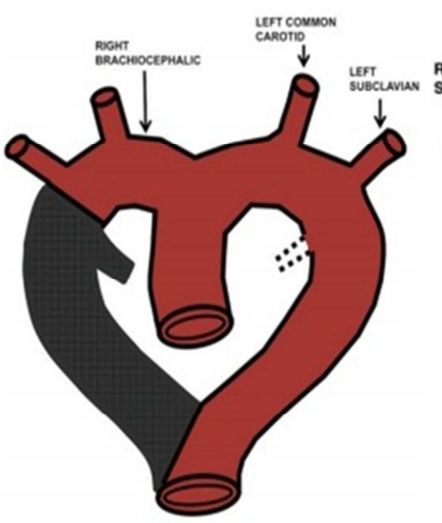

(b)

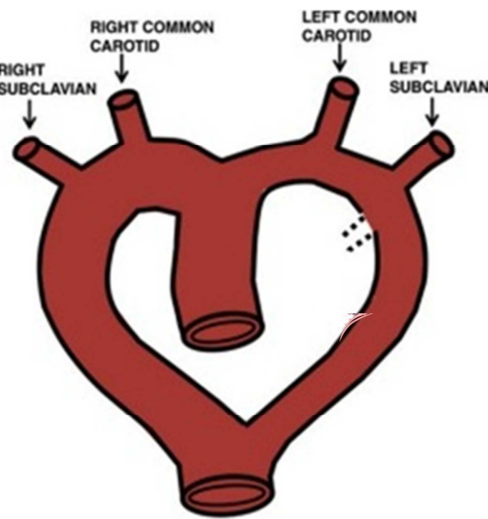

(c)

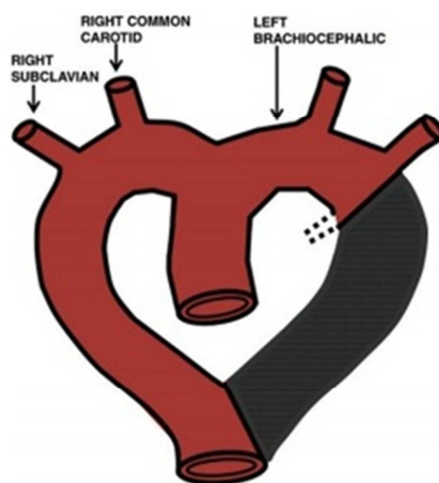

(d)

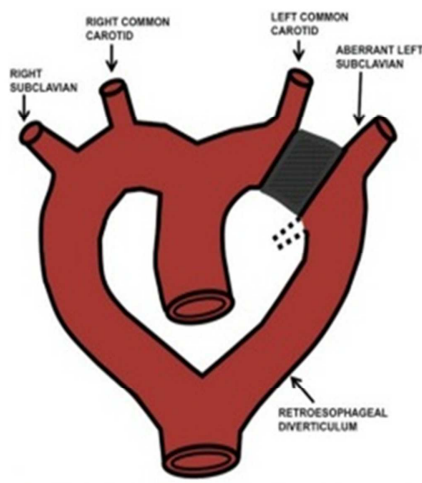

(e)

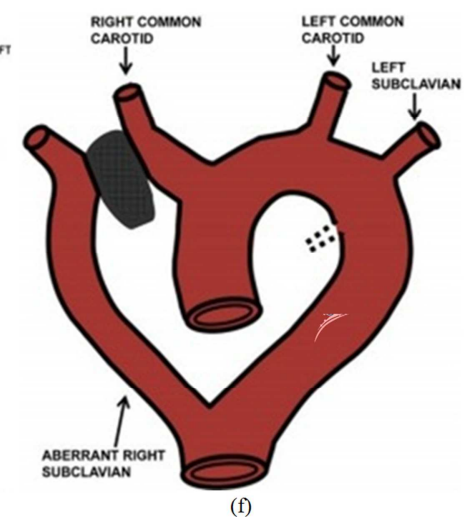

Figure 1. Embryonic 6 aortic arches and various rings [2]. (a) Schematic diagram depicting development of the embryonic 6pairs of aortic arches and branches. Gray areas represent segments that normally involute; (b) A normal left aortic arch which results from regression of the right arch. The first branch arising from the arch is the brachiocephalic artery, followed by the left common carotid and left subclavian arteries; (c) Hypothetical double aortic arch system with bilateral aortic arches and bilateral ductusarteriosi; (d) Right aortic arch with mirror image branching. This anomaly results from regression of the left arch between the LSC artery and the descending aorta. The first branch arising from the arch is the left brachiocephalic artery, followed by the RCC and then the RSC arteries; (e) Right aortic arch with aberrant left subclavian artery arising from a retroesophageal diverticulum of Kommerell. This anomaly results from regression of the left arch between the LCC and LSC arteries (gray), usually with persistence of the left sixth arch as ligamentumarteriosum; (f) Left aortic arch with aberrant right subclavian artery without diverticulum of Kommerell. This anomaly results from regression of the right arch between the RCC and RSC arteries, including the right ductusarteriosus (gray).
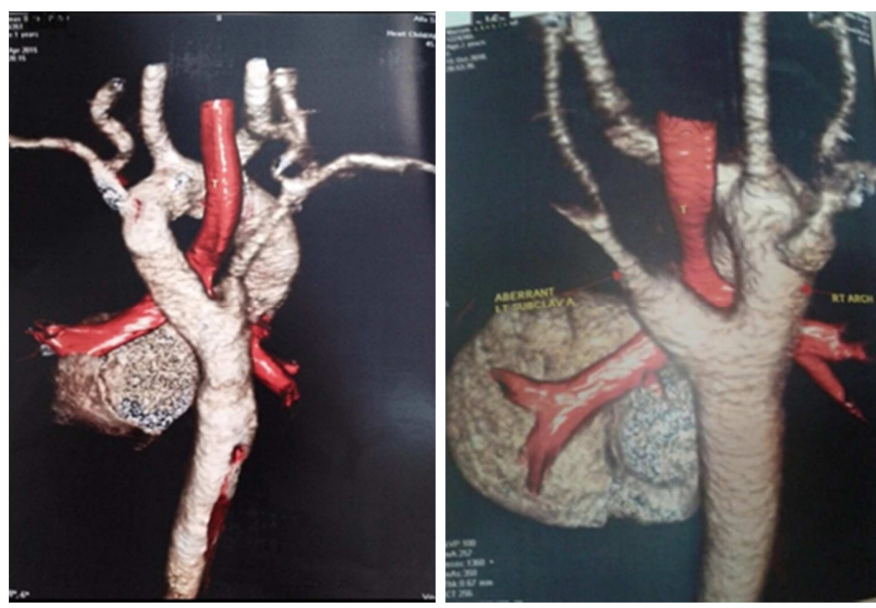

Figure 2. a MSCTA (frontal view, left image) done for the representative patient in our study population showing RAA with Left aberrant subclavian artery with tracheal compression, $b$ (back view, right image). 


\subsection{Data Collection}

Table 1. Showing types of vascular rings in our study.

\begin{tabular}{lll}
\hline Type of vascular ring & number & percentage \\
\hline DAA & 47 & $74.6 \%$ \\
Aberrant RT innominate & 1 & $1.6 \%$ \\
RAA & 10 & $15.9 \%$ \\
Pul. Art. Sling & 5 & $7.9 \%$ \\
\hline
\end{tabular}

Pertinent data extracted from their records either preoperative, intraoperative, and postoperative such as demographic data, presenting symptoms, findings in preoperative chest $\mathrm{x}$-ray (CXR), and computed tomography
(CT) and intra-operative correlation, surgical complications, and eventually the outcome (Table 1).

\subsection{Statistical Analysis}

Data analysis was carried out via using SPSS (Statistical Package for Social Science) version21. Qualitative data were represented in the form of mean \pm standard deviation. Continuous variable data were expressed as the mean \pm standard deviation. Statistical comparisons were made through Student's t-test for continuous variables and chisquare test for categorical variables. Results were considered statistically significant if P-value is less than 0.05 .

Table 2. Showing presenting symptoms in relation to different types of vascular rings.

\begin{tabular}{|c|c|c|c|c|c|}
\hline & DAA & RAA+LT Ligamentum & PulmonaryArtery sling & Aberrant RT innominate & P Value \\
\hline stridor & $24(51.1 \%)$ & $1(10 \%)$ & 0 & $1(100 \%)$ & 0.03 \\
\hline BA like symptoms & $22(46.8 \%)$ & $9(90 \%)$ & $4(80 \%)$ & 0 & 0.15 \\
\hline Feeding problems & $2(4.3 \%)$ & 0 & $5(100 \%)$ & $1(100 \%)$ & 0.001 \\
\hline
\end{tabular}

\subsection{Radiological Diagnosis}

Plain chest X-ray postero-anterior and lateral views were done in $100 \%$ as routine in the outpatient clinic. Upon suspecting or finding a right sided arch; patients were further investigated. No Barium swallow was used, due to inconvenience; especially in young infant or preschool children. Echo-Doppler examination $(88.9 \%)$ which was very helpful in diagnosing and defining which type of vascular ring. Bronchoscopy was used in 25 cases $(39.7 \%)$, done by pulmonologists before referral. Cardiac catheterization in only 2 patients (3.2\%). Multislice CT with $3 \mathrm{D}$ reconstruction was used in 59 patients $(93.7 \%)$. It is considered an excellent, non-invasive diagnostic modality. See table 3.

Table 3. Shows the diagnostic modalities used in our study.

\begin{tabular}{lll}
\hline Diagnostic modalities & Total $(\mathbf{n}=\mathbf{6 3})$ & Percentage \\
\hline ChestXray & 63 & $100 \%$ \\
Echo & 56 & $88.9 \%$ \\
Bronchoscope & 25 & $39.7 \%$ \\
Angiography & 2 & $3.2 \%$ \\
MSCT & 59 & $93.65 \%$ \\
\hline
\end{tabular}

\section{Results}

Patients' age in our study ranged from 2 months to 57 months (mean $16.25 \pm 1.3 \mathrm{~m}$ ), weight ranges from $3 \mathrm{~kg}$ to $26 \mathrm{~kg}$ (mean $9.87 \pm 0.8 \mathrm{~kg}$ ). Males were 38 cases $(60.3 \%$ ) and male: female ratio was $1.5: 1$. In table 1 various types of vascular rings were encountered. In our series, Double Aortic Arch (DAA) was right dominant arch variant in 25 (53.2\%) and left arch dominant in $7(14.9 \%)$ while the co-dominant variant was seen in 15 (31.9\%). Symptoms presented in our series were mostly due to tracheal compression in form of stridor, bronchial asthma, feeding problem, and failure to thrive shown in table 2. We depended on a variety of diagnostic methods in our series, mainly CXR in $100 \%$ see table 3. CXR findings like an absent left aortic knuckle, right-sided aortic arch, tracheal compression or indentation. Echocardiography with tissue Doppler assessment was used not only for confirming diagnosis but also helped in detecting 4 cases with associated cardiac anomalies (1 Fallot's tetralogy (1.6\%), 1 Patent ductus arteriosus (PDA) (1.6\%), 2 patent foramen ovale (PFO) $(3.2 \%)$.

Fifty-seven cases of vascular rings $(90.5 \%)$ operated by left posterolateral thoracotomy. Five cases of pulmonary artery sling $(7.9 \%)$ operated by median sternotomy and one case of innominate artery compression $(1.6 \%)$, no reported intraoperative complications nor intraoperative mortality in our study.

No injury happened to either phrenic or recurrent laryngeal nerve. One case was complicated with pneumothorax (1.6\%) and was treated by an intercostals tube. No bleeding in our cases as a complication. Three cases (4.7\%) were reventilated. Chylothorax happened in 4 cases $(6.3 \%)$ and managed by total parentral nutrition and fat intake restriction alone in one case $(25 \%)$ and in 3 cases $(75 \%)$, somatostatin was added but eventually, we went back to theatre for thoracic duct ligation in 2 cases $(50 \%)$.

We have only one case of mortality $(1.6 \%)$, pulmonary artery sling with diffuse narrowing of lower tracheal and this case died in the intensive care unit (ICU) $12 \mathrm{~h}$ early postoperatively. In our study, total postoperative stay ranged from (3-23 days).

Correlations through Q- square and student t-test revealed no significant association between the type of complication and type of vascular ring and age or weight, or gender, $\mathrm{P}$ value $>0.05$. Also, no significant association between ICU and ward stay and age or gender or presenting symptoms like stridor or asthma, $\mathrm{p}$ value $>0.05$.

There is a significant association between ward stay and types of vascular rings, $p$-value $<0.05$. Also, there is a significant association between ICU and ward stay and PostOperative complications, $\mathrm{p}$-value $<0.05$. 


\section{Discussion}

During fetal life, any anomaly associated with the development of the 6 pairs of aortic arches can lead to a vascular ring. Gross in 1945, introduced that term where a vascular anomaly encircles the aerodigestive tracts either completely or partially after he successfully relieved tracheal obstruction in a double aortic arch patient. Thus, they are classified as complete or partial; but both share the same symptoms and the same treatment algorithm. The double aortic arch (DAA) is by far the most common variant, seen in 57 cases $(74.6 \%)$ and this is similar to other reports from similar countries where the incidence of congenital heart diseases are near equal; they reported $68 \%$ and $56.3 \%$ incidence of DAA in 2 different reports $[1,3]$. The rightsided aorta with aberrant subclavian and left ligmentum is our second common type; representing $15.9 \%$ which is lower than most reports, where RAA represents $45 \%$ or $26.8 \%$ [4, 5]. The third common type in our study was pulmonary artery sling accounting for $7.9 \%$ and it's considered high if compared to Ronald`s report, it constitute 3.7\% [5].

Usually, the presenting symptoms of the incomplete vascular ring are severe and annoying; so patients present earlier. The severity of the symptoms is directly related to the degree of compression exerted by the rings. Because of this, complete rings present earlier versus incomplete rings. This is similar to other reported surgical series [6]. On the other hand, it could be totally asymptomatic and discovered during an investigation for other conditions.

The presence of right-sided aortic arch is a diagnostic clue in plain CXR; also, the lateral indentation of the tracheal air column. We have done CXR in $100 \%$ of our cases. Bronchoscopy was done in $39.7 \%$, findings were external compression, narrowing, and transmitted pulsation; but we no longer recommend except in severe dyspnea or stridor. But we think noninvasive radiologic imaging is enough to help and bronchoscope may cause laryngeal edema [4].

We used echocardiography principally due to its accuracy, simplicity and because of being non-invasive method, but in the presence of the highly specific and sensitive diagnostic MSCT, lately, we did not use echocardiography except if we suspect associated cardiac anomalies, exactly in 56 out of 63 cases $(88.9 \%)$. This nearly coincides with Shah and his colleagues, who used echocardiography in $95 \%$ in their study [7].

We had 4 cases with associated anomalies, Fallot tetralogy (1.6\%), PDA (1.6\%), PFO (3.2\%). Compared to Khalfan's study in 2006, the associated cardiac anomalies were found in 14 patients $(17 \%)$, ventricular septal defect in 10 patients $(12 \%)$, atrial septal defect in 4 patients $(5 \%)$, patent ductus arteriosus in 3 patients (4\%), and tetralogy of Fallot in 3 patients (4\%) [8].

In our study; cardiac catheterization was needed in $3.2 \%$ as an accurate method to delineate the anatomy but this was no longer used because of being an invasive technique and the need for contrast and replaced by Echocardiography and/or CT chest. Cardiac catheterization was used largely as in Khalfan`s series [8] that reported 33 (41\%) of 81 cases, also in another series of cardiac catheterization was used in 21 (47.7\%) from 44 cases [9].

Multislice CT is a noninvasive modality for diagnosis. It illustrates the arch dominance and tracheal compression. We used it in 59 cases, and our results match the results of many reportsin which all cases were diagnosed by contrastenhanced MSCT [10, 11]. On contrary, in Raghavan`s study, they used Magnetic resonant Imaging (MRI), but as we notice, this was a relatively old study [3]. However, we recommend MSCT for all our cases for better delineation and good decision for operation. The only disadvantage is radiation exposure. Our preference was based on the accessibility to that new imaging modality and the preference of the radiologist and surgeon interpreting the images as it provides an excellent correlation with intraoperative findings.

Stridor is the commonest presenting symptom among cases of complete vascular ring; mostly these cases were misdiagnosed as foreign body inhalation and referred for diagnostic bronchoscopy or sent for ENT evaluation. In our series, 26 of 63 cases $(41.3 \%)$ presented with stridor with 24 from the DAA group. Our results are comparable to other series with similar results $[3,7]$.

In our series, aerodigestive symptoms like dysphagia for solid foods or feeding troubles was seen in $(12.7 \%)$. Other studies reported higher percentage $27 \%$ and $31.3 \%[7,12]$.

Four of our patients had associated cardiac anomalies $(6.3 \%)$. Other series reported a higher incidence of cardiac anomalies as in the series but in our series, there were no associated non-cardiac anomalies compared to the same report, they documented respiratory anomalies in 13 patients $(40.6 \%)$ [12].

Our principle of operating as soon as possible in any case with a vascular ring was due to early repair allows normal trachea and esophagus growth. Similar to Yoon`s report in 2012, [12] we approached through a limited left posterolateral thoracotomy through the $3^{\text {rd }}$ intercostal space in $(92.1 \%)$, similar to others who reported $(91.8 \%)$ and $(92 \%)$ $[4,8]$.

Five sternotomies were used for cases with pulmonary artery sling. That allowed easy dissection and a wide non compromised field for re-implantation. In our series, no Video-assisted thoracoscopic surgery (VATS) was used in contrast to Rahul's report in 2007 who operated 39 cases using VATS (60.9\%) of 64 cases [7].

We tried our best to extubate as early as possible, on the operating table, or after transfer to the surgical intensive care unit to avoid upper airway edema. With pulmonary toilet, nasotracheal suction and chest percussion following extubation. Usually, better results are harvested and minimal postoperative atelectasis or pneumonia. We always inform and educate their parents that symptoms could persist up to six months before disappearing completely. In our 63 cases, postoperative stay ranges from ( 3 to 23 days), the extended stay was attributed to the development of chylothorax, and that coincides with a recent report where the mean hospital stay was (18.8 \pm 9.3$)$ days [13].

Mortality in our series was one case with pulmonary artery 
sling. The infant had diffuse narrowing of the lower trachea extending to the left main bronchus. We started to operate off-pump, then we had to go on by-pass emergently, it was complicated by difficult weaning from the ventilator as the stenosed tracheal segment mostly caused air trapping, $\mathrm{CO} 2$ narcosis, and death.

\section{Conclusion}

A vascular ring is a rare congenital cardiovascular anomaly that needs a high index of clinical suspicion for diagnosis. Operative strategy to minimize improper or incomplete management and prevent recurrence mainly relies on extensive dissection, delineation of the different components of the anomaly, and division of non-dominant arch and division of the ligamentum, freeing all fibrous bands between the arch and esophagus and trachea. For better results; we need collaboration from many specialists who will receive these patients before us as pediatricians and pulmonologists who must be aware of such anomaly with its multiple variants.

\section{Limitation of the Study}

This is a retrospective study that has the inherent deficiencies of this category of studies. Besides that, it is a single-center study, the number of cases is relatively small over a single decade period of time, with different variants of the same pathology. Data of follow up was needed but not documented in our files.

\section{List of Abbreviations}

VSD: Ventricular septal defect

CXR: Chest X-ray

CT: computed tomography

DAA: Double Aortic Arch

PDA: Patent ductus arteriosus

PFO: Patent foramen ovale

ICU: Intensive care unit

MRI: Magnetic resonant Imaging

\section{Declarations}

\section{Ethics Approval and Consent to Participate}

The study was approved by the Institutional Review Board (IRB) according to the code of ethics of the World Medical Association (Declaration of Helsinki) for studies involving humans, though the Committee's reference number was not applicable. The need for informed consent was waived due to the retrospective nature of the study.

\section{Consent for Publication}

Not applicable.

\section{Availability of Data and Material}

Data are available on request.

\section{Funding}

No external source of funding.

\section{Competing Interests}

No conflict of interestand nothing to disclose.

\section{References}

[1] Sanjeev Sharma, Jeri L Dobbs and Adnan Cobanoglu: Surgical Correction of Vascular Ring Anomalies. Asian Cardiovascular and Thoracic Annals, 2000; 8: 344.

[2] Hanneman K., Newman B., Chan F.: Congenital Variants and Anomalies of the Aortic Arch. Radio Graphics, 2017; 37 (1), 32-51.

[3] Raghavan Subramanyan, Poorthirikovil Venugopalan and Ravi Narayan: Vascular Rings: An important Cause of Persistent Respiratory Symptoms in Infants and Children. Indian Pediatrics, 2003; 40: 951-957.

[4] Backer CL, Constantine Mavroudis, Cynthia K. Rigsbyetal: Trends in vascular ring surgery. J Thorac Cardiovasc Surg, 2005; 129: 1339-1347.

[5] Ronald K. Woods, Ronald J. Sharp, George W. Holcombetal: Vascular anomalies and tracheoesophageal compression: a single institution's 25-yearexperience. Ann Thorac Surg, 2001; 72: $434-438$.

[6] Van Son JAM, Julsrud PR, Hagler D Jetal: Surgical treatment of vascular rings: the Mayo Clinic experience. Mayo Clin Proc, 1993; 68: 1056-1063.

[7] Rahul K. Shah, Bassem N. Mora, Emile Bachaetal: The presentation and management of vascular rings: An otolaryngology perspective. International Journal of Pediatric Otorhinolaryngology, 2007; 71: 57-62.

[8] Khalfan Alsenaidi, Rebecca Gurofskya, Tara Karamlouetal: Management and Outcomes of Double Aortic Arch in 81Patients, Pediatrics, 2006; 118: 1336.

[9] Mustafa Kir, Gul Sagin Saylam and Ulas Karadas: Vascular Rings: Presentation, Imaging Strategies, Treatment and Outcome, Pediatric Cardiology Media, 2012; 33: 607-617.

[10] Xin Chen, Yanjuan Qu and Zhi-Yuan Peng: Clinical value of multi-slice spiral computed tomography angiography and three-dimensional reconstruction in the diagnosis of double aortic arch. Exp Ther Med, 2014; 8: 623-627.

[11] Bai Song, LI Xiao-feng, Liu Cai-xiaetal: Surgical treatment for vascular anomalies and tracheoesophageal compression. Chin Med J, 2012; 125: 1504-1507.

[12] Yoon Jung Suh, Gi Beom Kim, Bo Sang Kwonetal: Clinical Course of Vascular Rings and Risk Factors Associated with Mortality. Korean Circ J, 2012; 42: 252-258.

[13] Song Zw, Xu Cy, Ge W.: The diagnostic value of MSCT multidimensional reconstructions for congenital vascular ring with tracheal stenosis. Zhonghua.

[14] Yi Xue Za Zhi, 2011; 91: 619-622. 